\title{
Online Appendix: Substitution and Stigma: Evidence on Religious Markets from the Catholic Sex-Abuse Scandal
}

\section{DANIEL M. HUNGERMAN}

This online appendix presents regression results referred to, but not shown, in the text.

The first 3 columns of Table 3

\begin{tabular}{|c|c|c|c|c|c|c|c|c|c|}
\hline & \multicolumn{3}{|c|}{ Per Capita Allegations: } & \multicolumn{3}{|c|}{ Census-Division Allegations } & \multicolumn{3}{|c|}{ Probit } \\
\hline & $\begin{array}{c}\text { On Catholic } \\
\text { Dummy }\end{array}$ & $\begin{array}{l}\text { On Not- } \\
\text { Catholic } \\
\text { Dummy }\end{array}$ & $\begin{array}{c}\text { On No Religion } \\
\text { Dummy }\end{array}$ & $\begin{array}{c}\text { On Catholic } \\
\text { Dummy }\end{array}$ & $\begin{array}{l}\text { On Not- } \\
\text { Catholic } \\
\text { Dummy }\end{array}$ & $\begin{array}{c}\text { On No Religion } \\
\text { Dummy }\end{array}$ & $\begin{array}{c}\text { On Catholic } \\
\text { Dummy }\end{array}$ & $\begin{array}{l}\text { On Not- } \\
\text { Catholic } \\
\text { Dummy }\end{array}$ & $\begin{array}{c}\text { On No Religion } \\
\text { Dummy }\end{array}$ \\
\hline Allegations & $\begin{array}{c}-0.00293 \\
{[0.00160]}\end{array}$ & $\begin{array}{c}-0.00004 \\
{[0.00226]}\end{array}$ & $\begin{array}{c}0.00297 \\
{[0.00215]}\end{array}$ & $\begin{array}{c}-0.00037 \\
{[0.00022]}\end{array}$ & $\begin{array}{l}-0.00014 \\
{[0.00016]}\end{array}$ & $\begin{array}{c}0.00051 \\
{[0.00028]}\end{array}$ & $\begin{array}{c}-0.00041 \\
{[0.00016]}\end{array}$ & $\begin{array}{c}-0.00003 \\
{[0.00012]}\end{array}$ & $\begin{array}{c}0.00054 \\
{[0.00025]}\end{array}$ \\
\hline
\end{tabular}
region, rather than the state. The last three columns redo the first three columns of Table 3 using Probit regressions rather than OLS (the coefficients are marginal effects). These coefficients are unadjusted (ie, they are not adjusted by a factor of 10).

Columns 4 through 6 of Table 3

\begin{tabular}{|l|c|c|c|}
\hline & \multicolumn{3}{|c|}{ Per Capita Allegations: } \\
\hline & $\begin{array}{c}\text { On Catholic } \\
\text { Dummy }\end{array}$ & $\begin{array}{c}\text { On Not-Catholic } \\
\text { Dummy }\end{array}$ & $\begin{array}{c}\text { On No Religion } \\
\text { Dummy }\end{array}$ \\
\hline Allegations & -0.00061 & -0.00004 & 0.00065 \\
& {$[0.00015]$} & {$[0.00013]$} & {$[0.00017]$} \\
\hline Future Allegations & -0.00005 & -0.00019 & 0.00024 \\
(2-Year Lead) & {$[0.00017]$} & {$[0.00017]$} & {$[0.00015]$} \\
\hline
\end{tabular}

These three columns redo columns 4-6 of Table 3, using only the 2-year-lead allegation coefficient. Coefficients are unadjusted (ie, they are not adjusted by a factor of 10)

Table 6

\begin{tabular}{|c|c|c|c|c|c|c|c|c|c|}
\hline & $\begin{array}{l}\text { Donations: } \\
\text { Dropping } \\
\text { Imputations }\end{array}$ & $\begin{array}{l}\text { Per Capita } \\
\text { Allegations: } \\
\text { Membership }\end{array}$ & $\begin{array}{l}\text { Per Capita } \\
\text { Allegations: } \\
\text { Donations }\end{array}$ & $\begin{array}{l}\text { With 2001/02: } \\
\text { Membership }\end{array}$ & $\begin{array}{c}\text { With 2001/02: } \\
\text { Donations }\end{array}$ & $\begin{array}{l}\text { Allegations } \\
\text { from prior year: } \\
\text { Membership }\end{array}$ & $\begin{array}{l}\text { Allegations } \\
\text { from prior year: } \\
\text { Donations }\end{array}$ & $\begin{array}{c}\text { Current } \\
\text { Weights: } \\
\text { Membership }\end{array}$ & $\begin{array}{c}\text { Current } \\
\text { Weights: } \\
\text { Donations }\end{array}$ \\
\hline Allegations & $\begin{array}{c}0.00257 \\
{[0.00097]}\end{array}$ & $\begin{array}{c}0.00272 \\
{[0.00188]}\end{array}$ & $\begin{array}{c}0.00289 \\
{[0.00823]}\end{array}$ & $\begin{array}{c}0.00040 \\
{[0.00022]}\end{array}$ & $\begin{array}{c}0.00229 \\
{[0.00081]}\end{array}$ & $\begin{array}{c}0.00038 \\
{[0.00027]}\end{array}$ & $\begin{array}{c}0.00141 \\
{[0.00068]}\end{array}$ & $\begin{array}{c}0.00031 \\
{[0.00023]}\end{array}$ & $\begin{array}{c}0.00238 \\
{[0.00094]}\end{array}$ \\
\hline
\end{tabular}

Column 1 redoes column 2 of table 6 dropping imputed donations data. Columns 2 and 3 redo columns 1 and 2 of Table 6 using per-capita allegations instead of total allegations. Columns 4 and 5 redo columns 1 and 2 of Table 6 adding back in data from the year 2001/02. Columns 6 and 7 redo the columns 1 and 2 in Table 6 using allegations from the prior year, instead of the current year. The last

two columns redo columns 1 and 2 in Table 6 weighting by current membership (rather than 1990 membership). Coefficients are unadjusted (ie, they are not adjusted by a factor of 10). 
Columns 3 and 4 of Table 6

\begin{tabular}{|l|c|c|}
\hline & On Membership & On Donations \\
\hline Allegations & 0.00070 & 0.00143 \\
& {$[0.00029]$} & {$[0.00069]$} \\
\hline Future Allegations & -0.00060 & 0.00018 \\
(2-Year Lead) & {$[0.00023]$} & {$[0.00030]$} \\
\hline
\end{tabular}

